\title{
Evaluation of a culture slide in the diagnosis of vaginal candidosis
}

\author{
R S PATTMAN, M S SPROTT, AND T R MOSS \\ From the Department of Sexually Transmitted Diseases and the Public Health Laboratory, Newcastle \\ General Hospital, Newcastle upon Tyne
}

SUMMARY The Till-U-Test Candida Dermatophyte (TUT CD) culture slide, produced for the diagnosis of yeast and dermatophyte infections, was compared with microscopy and formal laboratory culture in the diagnosis of vaginal candidosis. Candida albicans grew readily on the medium and reliable results were obtained within a mean of three days' incubation at room temperature. Agreement with laboratory culture was $91 \cdot 4 \% ; 29 \%$ of cases would have been missed by microscopy alone. The TUT CD is a useful device, therefore, in the investigation of vaginitis.

\section{Introduction}

Recent reports indicate an increasing prevalence of vaginal candidosis in women attending clinics for the treatment of sexually transmitted diseases, amounting to 129 per 100000 population in England between 1974 and $1978 .^{1}$ Candidosis was diagnosed in $19 \%$ of women attending these clinics in $1978 .{ }^{1}$ In a study of 213 patients attending their general practitioners because of symptomatic vaginal discharge or pruritus vulvae $48 \%$ had vaginal yeast infection. $^{2}$

Diagnosis may be made by examination of vaginal material directly using darkground microscopy or after staining by Gram's method. ${ }^{3}$ This can be confirmed by culture on a selective medium and isolates identified as Candida albicans by germ tube formation, fermentation, assimilation, and agglutination tests. ${ }^{4}$ Microscopy, depending on the experience of the observer, is relatively insensitive as only $36-52 \%$ of cases are correctly diagnosed. ${ }^{5-8}$

The aim of this study was to evaluate a new dipslide, Till-U-Test Candida Dermatophyte (TUT CD, Tillomed Laboratories Ltd), for the culture of dermatophytes and yeasts in the clinic or surgery. The slide consists of a modified Sabouraud's medium on one face and a selective differential medium for common dermatophyte species of Trichophyton, Microsporum, and Epidermophyton on the other.

\footnotetext{
Address for reprints: Dr R S Pattman, Department of Sexually Transmitted Diseases, Ward 34, Newcastle General Hospital, Westgate Road, Newcastle upon Tyne, NE4 6BE
}

Accepted for publication 6 July 1980

\section{Patients and methods}

STUDY POPULATION

One hundred and fifty-four women attending the department of sexually transmitted diseases at Newcastle General Hospital were studied. Their mean age was 24.6 years (range 16-48 years). The first 60 patients were unselected and seen consecutively while the remaining 94 either had symptoms of vaginitis (altered discharge or pruritus vulvae) or were contacts of male patients with balanitis or nongonococcal urethritis.

\section{DIAGNOSIS AND IDENTIFICATION}

A history was taken with special reference to the use of antibiotics and contraception during the preceding month.

Gram-stained smears of material from the urethra, cervix, and vagina were studied for Gram-negative diplococci and yeasts, and a saline suspension of material from the vagina was examined by darkground microscopy for Trichomonas vaginalis and yeasts.

Material from the urethra and cervix was cultured on selective medium for Neisseria gonorrhoeae, from the cervix for Chlamydia trachomatis, and from the vagina for the identification of $T$ vaginalis. Swabs from the vaginal walls were inoculated on to Sabouraud's glucose agar and incubated at $37^{\circ} \mathrm{C}$ for 48 hours.

Morphologically typical colonies were identified as C albicans by germ-tube formation and agglutination tests with $C$ albicans antiserum. Further vaginal samples were inoculated on to both faces of a TUT 
CD slide and incubated at room temperature in the clinic for one week. The slides were examined every 24 hours. Positive slides were identified by the presence of characteristic "off-white" colonies on the modified Sabouraud's medium and "putty-like" colonies on the dermatophyte medium. A final diagnosis of candidosis was made only if positive results were obtained from at least two of the three tests.

\section{Results}

Vaginal candidosis was diagnosed in 35 women with a mean age of 21.9 years (range 18-40 years), four of whom were contacts of men with nongonococcal urethritis. Sixty (39\%) women in the study were using a combined oral contraceptive preparation and this included $13(37 \%)$ of those with candidosis. Five patients found to have candidosis had received antibiotics within the preceding month.

\section{MICROSCOPY}

Candidosis was provisionally diagnosed by microscopy in 27 cases and confirmed in only 25 of these by one or both culture methods. The two falsely diagnosed patients were menstruating and were also found to be infected with $T$ vaginalis.

\section{CULTURE}

Reliable interpretation of the culture slide could be made after a mean of 2.8 days (range 1-6 days). Characteristic multiple colonies (figure) were found on both faces of 32 culture slides, with less than five colonies on the other three. One TUT CD slide produced typical colonies in the absence of laboratory or microscopical evidence of infection. $C$ albicans was isolated and identified on Sabouraud's glucose agar in 33 cases, although growth was scanty in those cases in which less than five colonies were found on the slides. Laboratory culture alone gave a positive result in one case with microscopical evidence of yeast infection. In two cases with positive results by microscopy and TUT CD slides, there was no growth on the Sabouraud's medium after $\mathbf{4 8}$ hours' incubation. Both culture methods gave positive results in 32 $(91.4 \%)$ of the 35 patients with candidosis. Atypical clear colonies were seen on one TUT CD slide and Escherichia coli was identified after subsequent laboratory subculture.

Trichomoniasis was diagnosed in 24 women, gonorrhoea in 18, and infection with $C$ trachomatis in 15 , nine of whom were contacts of men with nongonococcal urethritis.

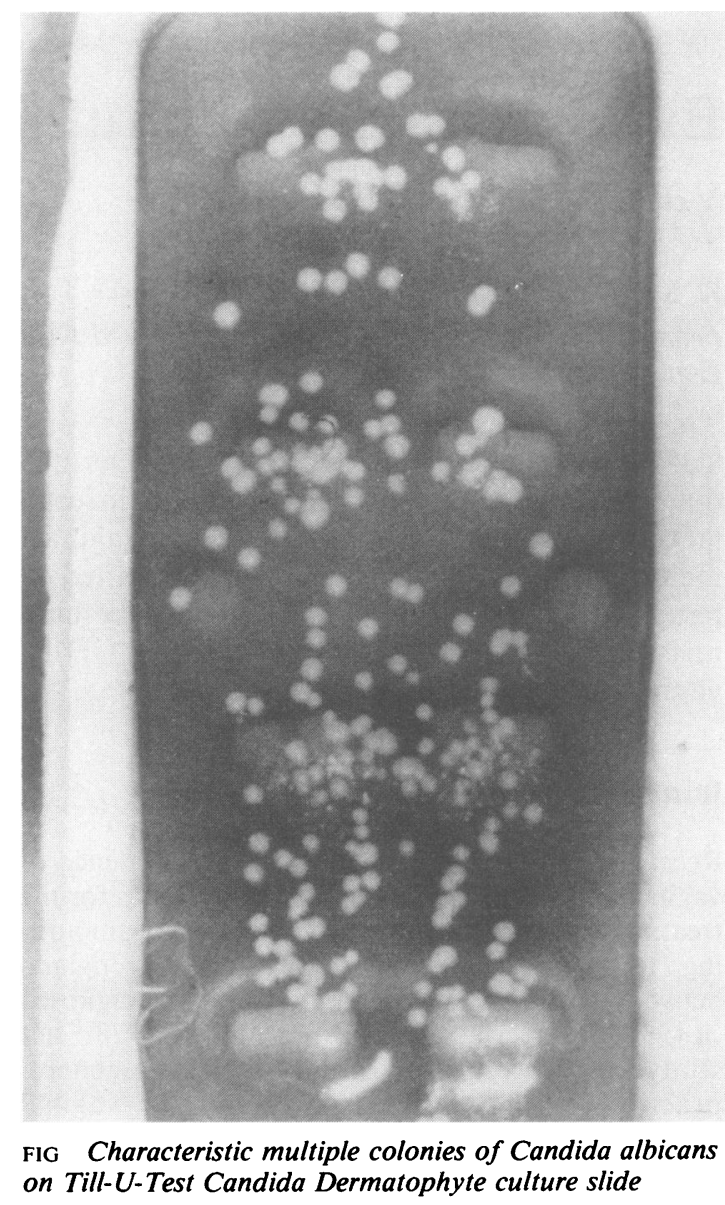

\section{Discussion}

Diagnosis by culture slide was easy and reliable; interpretation was possible within three days in most cases after incubation at room temperature. Agreement with laboratory culture was excellent, with positive results for both in $91.4 \%$ of patients. Weissberg, using a similar dip-slide preparation, achieved agreement in $96 \%$ of cases with conventional Sabouraud's medium, although incubation at $37^{\circ} \mathrm{C}$ was required. ${ }^{9}$

Ten $(29 \%)$ cases would have been missed if microscopy alone had been relied upon. This is a smaller proportion than previously quoted and may be partly explained by the fact that most cases reported here had both wet films and Gram-stained samples examined whereas previous studies have only compared one of these techniques with culture. Only one false-positive culture result was obtained. 
Concurrent oral contraception appeared to be unrelated to the finding of candidosis, although the figures were small.

Vulvovaginitis is a common and often distressing complaint, the cause of which may not be correctly identified on clinical and microscopical examination. In $35 \%$ of clinics for the treatment of sexually transmitted diseases in England a diagnosis of candidosis is made by microscopy alone. ${ }^{10}$ The TUT CD slide, therefore, is a useful diagnostic tool for a condition in which treatment is not determined by exact mycological identification of the pathogen. The expense of using this product may be counterbalanced by a reduction in the cost of unnecessary prescriptions and a reduced demand for laboratory services.

We would like to thank our medical colleagues and Sister Bulens with her nursing staff for helping to collect the specimens and Tillomed Laboratories Ltd for supplying the slides.

\section{References}

1. Department of Health and Social Security. Sexually transmitted diseases. On the State of the Public Health. Annual Report of the Chief Medical Officer of the Department of Health and Social Security for the Year 1978. London: HMSO. 1979:60-5.
2. Wright $\mathrm{HJ}$, Palmer A. The prevalence and clinical diagnosis of vaginal candidosis in non-pregnant patients with vaginal discharge and pruritus vulvae. $J R$ Coll Gen Pract 1978; 28:719-23.

3. Cowan ST, Steel KJ. In: Cowan ST, ed. Manual for the Identification of Medical Bacteria. 2nd ed. Cambridge: Cambridge University Press, 1975; 163.

4. Odds FC. Candida and Candidosis. 1 st ed. Leicester: Leicester University Press, 1979:42-7.

5. Willmott FE. Genital yeasts in female patients attending a VD clinic. Br J Vener Dis 1975; 51:119-22.

6. Thin RNT, Atia W, Parker JDJ, Nicol CS, Cant G. Value of Papanicolaou-stained smears in the diagnosis of trichomoniasis, candidiasis, and cervical herpes simplex virus infection in women. Br J Vener Dis 1975;51:116-8.

7. Becker H, Schweisfurth R. Untersuchungen über ein Medium zur Diagnostik der vaginalea Candidosis. Mykosen 1971; 14: 127-30.

8. Eddie DAS. The laboratory diagnosis of vaginal infections caused by Trichomonas and Candida (monilia) spores. J Med Microbiol 1968; 1: 153-9.

9. Weissberg SM. Evaluation of a dipstick for Candida. Obstet Gynaecol 1977; 52: 506-9.

10. O'Connor BH, Adler MW. Current approaches to the diagnosis, treatment, and reporting of trichomoniasis and candidosis. $\mathrm{Br} J$ Vener Dis 1979;55:52-7. 